\title{
Structure-anticancer activity relationships among 4-azolidinone-3-carboxylic acids derivatives
}

\author{
D. V. Kaminskyy, R. B. Lesyk \\ Danylo Halytsky Lviv National Medical University \\ 69, Pekarska, Lviv, Ukraine, 79010 \\ dr_r_lesyk@org.lviv.net; dankaminskyy@gmail.com
}

\begin{abstract}
The aim of present research was investigation of anticancer activity of 4-azolidinone-3-carboxylic acids derivatives, and studies of structure-activity relationships (SAR) aspects. Methods. Organic synthesis; spectral methods; anticancer screening was performed according to the US NCI protocol (Developmental Therapeutic Program). Results. The data of new 4-thiazolidinone-3-alkanecarboxylic acids derivatives in vitro anticancer activity were described. The most active compounds which belong to 5-arylidene-2,4thia(imida)zolidinone-3-alkanecarboxylic acids; 5-aryl(heteryl)idenerhodanine-3-succinic acids derivatives were selected. Determination of some SAR aspects which allowed to determine directions in leadcompounds structure optimization, as well as desirable molecular fragments for design of potential anticancer agents based on 4-azolidinone scaffold were performed. 5-Arylidenehydantoin-3-acetic acids amides were identified as a new class of significant selective antileukemic agents. Possible pharmacophore scaffold of 5-ylidenerhodanine-3-succinic acids derivatives was suggested. Conclusions. The series of active compounds with high anticancer activity and/or selectivity levels were selected. Some SAR aspects were determined and structure design directions were proposed.
\end{abstract}

Keywords: 4-azolidinone-3-carboxylic acids, anticancer activity, SAR.

Introduction. Design of «small molecules» as innovative anticancer agents based on well-known scaffolds is one of the most commonly employed approaches in drug discovery. Nowadays the anticancer potential of 4-azolidinone-3-carboxylic acids (derivatives of rhodanine, 2,4-thiazolidindione or hydantoin with carboxylic acids in position N3) realized via influence on various metabolic pathways of cancer cells is described based on traditional and high-throughput screening [1, 2] (Fig. 1). The large group of mentioned heterocycles with known pharmacological activity (such as antioxidant, anti-inflammatory, hypoglycemic, immunomodulative, etc.) was established as promising anticancer agents as well. Another approach to search of anticancer substances is target-oriented drug design, which

(C) Institute of Molecular Biology and Genetics NAS of Ukraine, 2010 allows to identify anticancer hit-compounds among 4-azolidinone-3-carboxylic acids derivatives [6]. Consequently, the row of 4-azolidinone-3-carboxylic acids derivatives with high affinity to "anticancer biotargets» was discovered. 5-Arylidenerhodanine-3-carboxylic acids are known as inhibitors of antiapoptic protein-protein interaction between Bcl-2 and Bax family and their interaction with receptors' domains [7-10]; inhibitors of JSP-1 - «atypical» dual-specific phosphatases family member (JNK-stimulating phosphatase-1) [11]. Among 4-azolidinone-3-carboxylic acids COX inhibitors are selected [12] because of their potential anticancer activity [6]. Hydantoin-carboxylic acids are inhibitors of Ras farnesyl transferase [13] and are considered to be perspective anticancer agents [14]. The ligands of neuroimunofiline FK506-binding protein (FKBP) are structurally analogous to mentioned series 

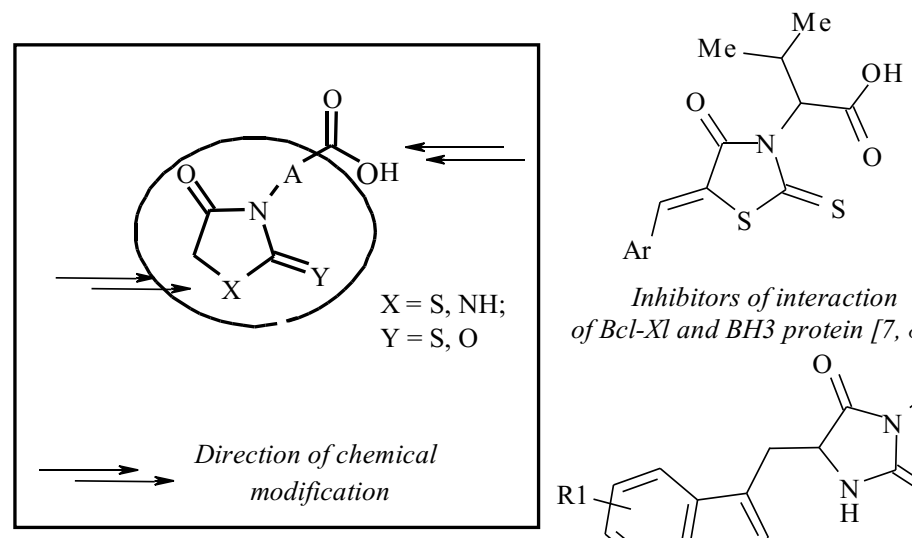

Inhibitors of interaction of Bcl-Xl and BH3 protein $[7,8]$

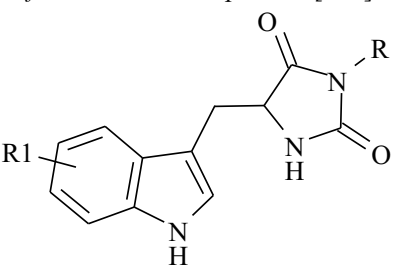

Necroptosis inhibitors [24]

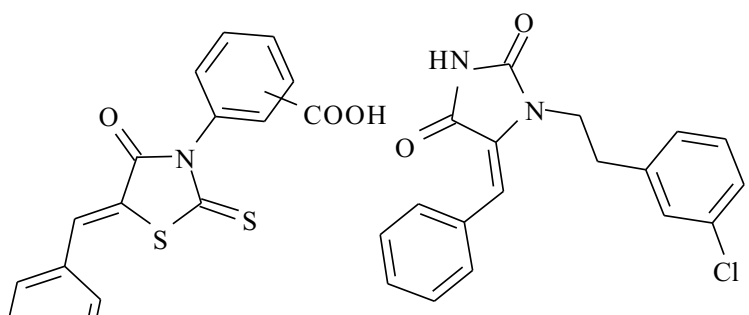

JSP-1 inhibitors [11]

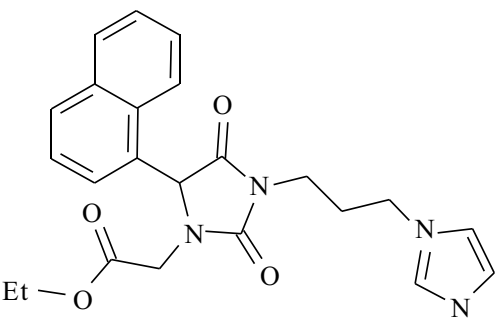

Farnesyl tranferase inhibitor [12]

Fig. 1. Structure of 4-azolidinone derivatives with anticancer activity

of compounds. Functionalized 4-imidazolidinone-3alkancarboxylic acids belong to a new group of noncovalent inhibitors of Human Leukocyte Elastase [16] .

There is known antiproliferative potential of 5substituted N-derivatives of hydantoin [17] and 5-arylidene-2,4-imidazolidinediones [18], which are related to EGFR-kinase (epidermal growth factor receptor) inhibition [19-21]. Among amides of latter acids the inhibitors of cyclin-depended kinase (CDK2/Cyclin A) [22] are selected. One of the discovered molecular mechanisms of 4-azolidinone-3-carboxylic acids is antagonism to $\alpha_{v} \beta_{3}$-receptors. Inhibitor possibility was improved based on correlation between expression factor progression and cancer development [23].

Also anticancer activity of 5-substituted 4-azolidinone-carboxylic acids are associated with p53 dependent pathways of apoptotic and neoplastic transformation [24] and inhibition of necroptosis (regulated caspase-independent cell death mechanism) [25]. Extensive research has been directed towards hypoxiabased strategies for anticancer agents [6]. 5-Ylidenerhodanines, which may be interpreted as synthetic precursors of rhodanine-3-carboxylic acids, modulate proliferation and apoptosis of cancer cells via influence on NO-related pathways [5]. There is an interesting fact of combined anti-inflammatory, antioxidant, and other related activities established for some compounds $[26$,
27], which is extremely important within the classical progression triad: stress-inflammation-cancer.

In the light of search for new 4-azolidinone-3carboxylic acids derivatives as potential anticancer agents the present work was aimed to investigation of anticancer activity of newly synthesized compounds and studies of some structure-activity relationships aspects.

Materials and methods. For anticancer activity screening the library of 4-azolidinone-3-carboxylic acids was previously synthesized [1, 28-30] using known synthetic methods. Synthetic approaches to target compounds differed and depended on nature of core heterocycles (rhodanine, 2,4-thiazolidinedione, hydantoin). Compounds of rhodanine row were synthesized by modification of rhodanine-3-carboxylic acids. Derivatives of acids belonged to 2,4-thia(imida)zolidinedione series were synthesized on the assumption of 2,4thiazolidinedione or hydantoin rings. 5-Ylidenederivatives of mentioned compounds were obtained by different modifications of Knoevenagel reaction (Fig. 2).

The structures and purity of synthesized compounds were elucidated by spectral data $\left({ }^{1} \mathrm{H}\right.$ NMR, IR, EIMS, LC-MS).

Newly synthesized compounds were selected by the National Cancer Institute (NCI) Developmental Therapeutic Program (www.dtp.nci.nih.gov) for the in 


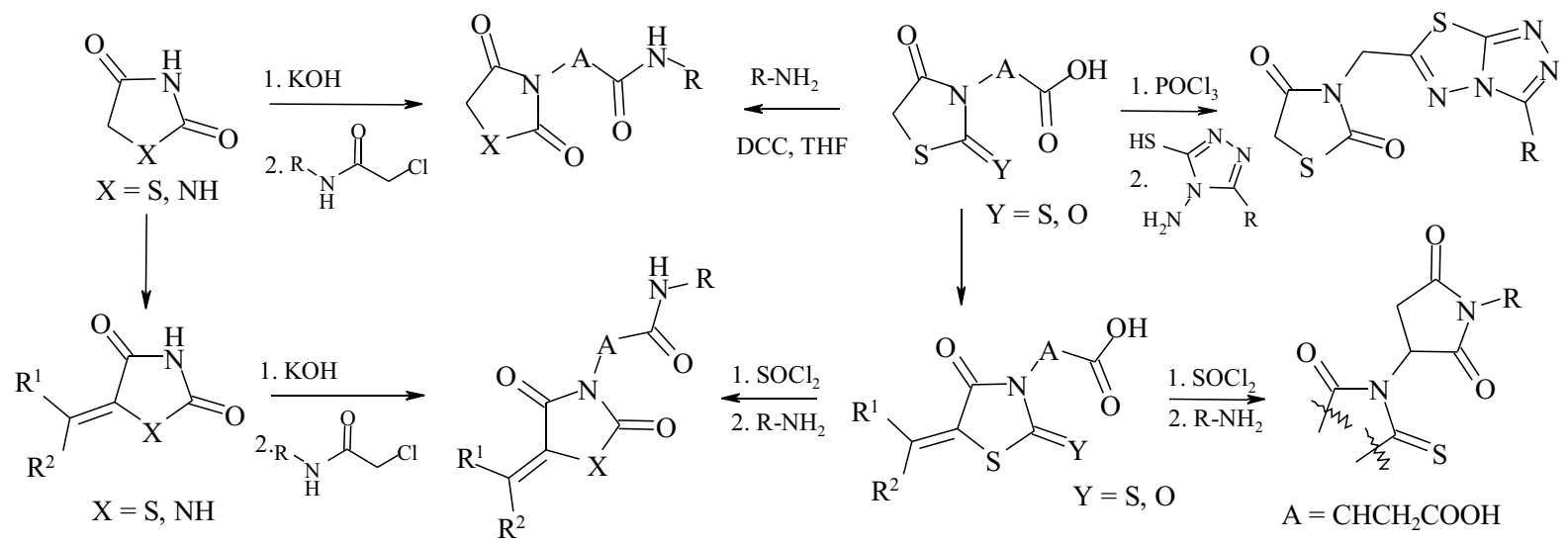

$\mathrm{R}^{1}=\mathrm{H} ; \mathrm{R}^{2}=\mathrm{Ar}$, Het, PhCHCH$; \mathrm{R}^{1}=\mathrm{R}^{2}=\mathrm{CH}_{3} ; \mathrm{R}^{1}+\mathrm{R}^{2}=\left(\mathrm{CH}_{2}\right)_{n} ; \mathrm{R}=\mathrm{Ar} ;\left(\mathrm{CH}_{2}\right)_{n} \mathrm{COOH} ; \mathrm{A}=\left(\mathrm{CH}_{2}\right)_{n}$

Fig. 2. General synthetic scheme

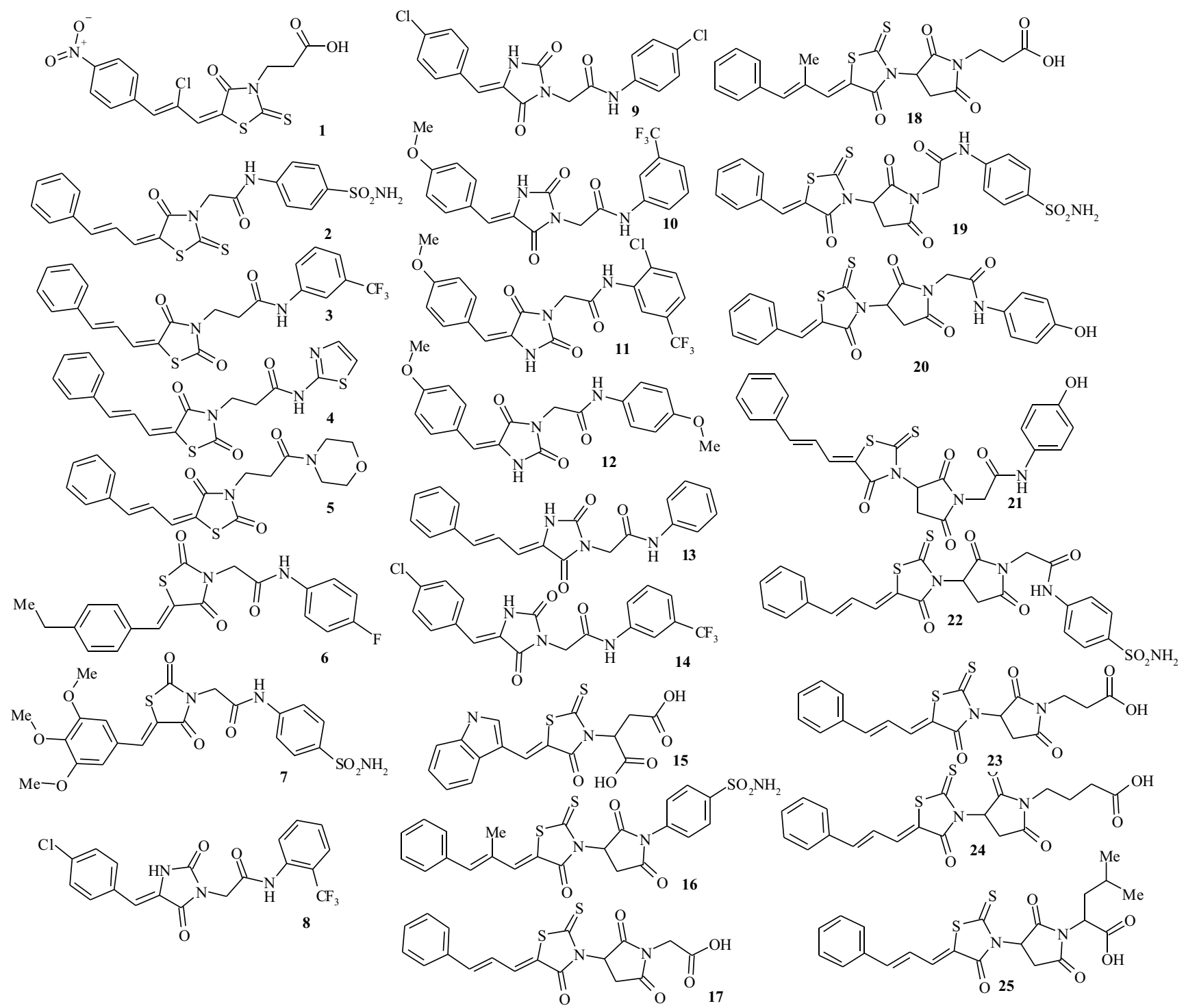

Fig. 3. Structure of selected samples for advanced anticancer screening (against the full panel of about 60 human tumor cell lines at 10 -fold dilutions of five concentrations ranging from $10^{-4}$ to $10^{-8} \mathrm{M}$ ) 
vitro cell line screening to investigate their anticancer activity. Anticancer assays were performed according to the US NCI protocol as described elsewhere [3134]. The compounds were first evaluated at one dose primary anticancer assay towards three cell lines (panel consisting of three types of human cancers: breast (MCF7), lung (NCI-H460) and CNS (SF-268) - concentration $10^{-4} \mathrm{M}$ ) or towards approximately 60 cell lines (concentration $10^{-5} \mathrm{M}$ ). The human tumor cell lines were derived from nine different cancer types: leukemia, melanoma, lung, colon, CNS, ovarian, renal, prostate and breast cancers. In the screening protocol, each cell line was inoculated and pre-incubated for $24-48 \mathrm{~h}$ on a microtiter plate.

Test agents were then added at a single concentration and the culture was incubated for further $48 \mathrm{~h}$. End point determinations were made with a protein binding dye, sulforhodamine B (SRB). Results for each test agent were reported as the percent growth of the treated cells when compared to the untreated control cells. A $48 \mathrm{~h}$ continuous drug exposure protocol was used with a SRB protein assay to estimate cell viability and growth.

The cytotoxic and/or growth inhibitory effects of the most active selected compounds were tested in vitro against the full panel of about 60 human tumor cell lines at 10 -fold dilutions of five concentrations ranging from $10^{-4}$ to $10^{-8} \mathrm{M}$. Using the seven absorbance measurements [time zero $(\mathrm{Tz})$, control growth in the absence of drug (C), and test growth in the presence of drug at the five concentration levels (Ti)], the percentage growth was calculated at each of the drug concentrations levels. Percentage growth inhibition was calculated as:

$[\mathrm{Ti}-\mathrm{Tz} / \mathrm{C}-\mathrm{Tz}] \cdot 100$ for concentrations for which $\mathrm{Ti} \geq \mathrm{Tz}$; $[\mathrm{Ti}-\mathrm{Tz} / \mathrm{Tz}] \cdot 100$ for concentrations for which $\mathrm{Ti}<\mathrm{Tz}$.

Three dose response parameters were calculated for each compound. Growth inhibition of $50 \%\left(\mathrm{GI}_{50}\right)$ was calculated from $[(\mathrm{Ti}-\mathrm{Tz}) /(\mathrm{C}-\mathrm{Tz})] \cdot 100=50$, which is the drug concentration resulting in a $50 \%$ lower net protein increase in the treated cells (measured by SRB staining) as compared to the net protein increase seen in the control cells. The drug concentration resulting in total growth inhibition (TGI) was calculated from $\mathrm{Ti}=$ $=\mathrm{Tz}$. The $\mathrm{LC}_{50}$ (concentration of drug resulting in a
$50 \%$ reduction in the measured protein at the end of the drug treatment as compared to that at the beginning) indicating a net loss of cells following treatment was calculated from $[(\mathrm{Ti}-\mathrm{Tz}) / \mathrm{Tz}] \cdot 100=-50$. Values were calculated for each of these three parameters if the level of activity is reached; however, if the effect was not reached or was exceeded, the value for that parameter was expressed as greater or less than the maximum or minimum concentration tested. The $\operatorname{lgGI}_{50}$, $\lg \mathrm{TGI}$, $\operatorname{lgLC}_{50}$ were then determined, defined as the mean of the log's of the individual $\mathrm{GI}_{50}, \mathrm{TGI}, \mathrm{LC}_{50}$ values. The lowest values are obtained with the most sensitive cell lines. Furthermore, mean graph midpoints (MG_MID) were calculated for each of the parameters, giving an average activity parameter over all cell lines for each compound. For the calculation of the MG_MID, insensitive cell lines were included with the highest concentration tested.

Results and discussion. During the first step of screening (using one concentration) on 3 cancer cell lines (MCF7, NCI-H460, SF-268) 49 compounds were tested. For mentioned samples different levels of antimitotic activity were established, thought in the majority of cases the maximum influence was observed against NCI-H460 line Non-small cell lung cancer line. 32 Compounds were tested using 60 cancer cell lines panel. Average values of panel cancer lines growth percent lay within $100 \%$, which provides evidence that nonspecific antimitotic action for studied 4-thiazolidinone derivatives. However, tested compounds possessed specific influence on some individual cell lines without influencing others. This fact may be related to effect on some metabolic pathways or biotarget of certain cell lines (www.dtp.nci.nih.gov; http://www.lg cpromochem-atcc.com). UO-31 and 786-O renal cancer cell lines have been found to be the most sensitive to testing compounds. For example 3-[3-(3-trifluoromethylphenyl)-[1,2,4]-triazolo-[3,4- $b][1,3,4]$-thiadiazol-6-yl-methyl]-thiazolidine-2,4-dione, 2-[2,4-dioxo5-(3,4,5-trimethoxybenzylidene)-thiazolidin-3-yl]-N(4-sulfamoylphenyl)-acetamide, 3-[5-(4-methoxybenzylidene)-4-oxo-2-thioxothiazolidin-3-yl]-1-(3-trifluoromethylphenyl)-pyrrolidine-2,5-dione and 2-(4-benzylidene-2,5-dioxoimidazolidin-1-yl)-N-(4-chlorophenyl)-acetamide provided not only growth inhibition but also death of UO-31 cells. The same effect was ob- 
Summary of anticancer activity of the compounds in different concentrations $\left(10^{-4}-10^{-8} \mathrm{M}\right)$ towards 60 cancer cell lines

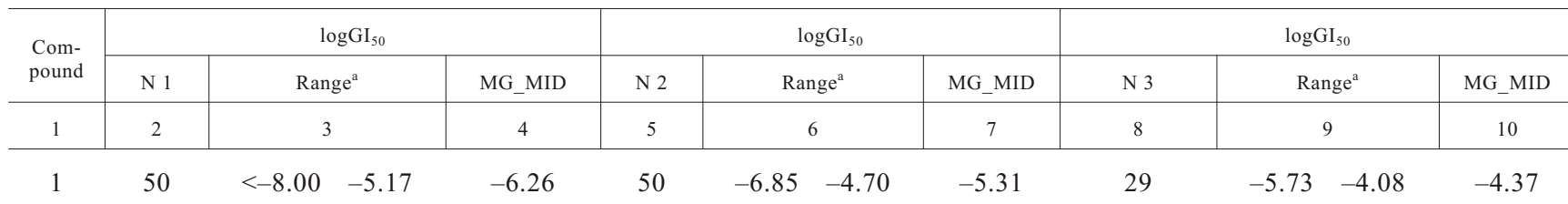

Most sensitive cell lines ( $\left.\log \mathrm{GI}_{50} / \log \mathrm{TGI}\right)$ : MOLT-4 -7,80/-6,85; SR $<-8,00 /-6,85$ (L); SW-620 <-8,00/-6,57 (CC); SF $539<-8,00 /-6,57$ (CNS)

$\begin{array}{llllllllll}2 & 28 & -5.22 \div-4.36 & 4.60 & 9 & -4.63 \div>-4.30 & -4.34 & - & >-4.30 & 4.30\end{array}$

Most sensitive cell lines $\left(\log \mathrm{GI}_{50} / \log \mathrm{TGI}\right)$ : HL-60 (TB) -5.22/-4.32; K-562 -5.16/-4.59; RPMI-8226 -5.10/-4.47 (L); NCI-H460 -5.20/-4.49; NCI-H522 -5.12/> -4.30 (NSCLC); KM12 -5.07/> -4.30 (CC); LOX IMVI -5.00/-4.51 (M); OVCAR-3 -5.14/-4.50 (OC); MDA-MB-435 -5.02/-4.51 (BC)

Most sensitive cell lines $\left(\log \mathrm{GI}_{50} / \log \mathrm{TGI}\right)$ : A549/ATTC $-5.41 />-4.30$; NCI-H460 -5.30/> -4.30 (NSCLC); HCT-116 -5.38/> -4.30 (CC); U251 -5.36/> -4.30 (CNS); SK-MEL5 -6.21/-5.39 (M); ACHN -5.08/> -4.30; $\mathrm{SN} 12 \mathrm{C}-5.20 />-4.30(\mathrm{RC}) ; \mathrm{MCF}-7-5.06 />-4.30 ; \mathrm{MDA}-\mathrm{MB}-231 / \mathrm{ATTC}-5.19 />-4.30(\mathrm{BC})$

Most sensitive cell lines ( $\left.\log \mathrm{GI}_{50} / \log \mathrm{TGI}\right)$ : CCRF-CEM -4.95/> -4.30 (L); SF $539-5.88 /-5.53$; U251 -5.34/-4.74 (CNS); LOX IMVI -5.10/-4.48; SK-MEL2 -5.25/-4.52; UACC62 -5.02/-4.46 (M); OVCAR-8 -5.05/> -4.30 (OC); ACHN -5.00/-4.45; UO31 -5.01/-4.36 (RC); DU-145 -5.03/> -4.30 (PC); MDA-MB-231/ATTC -5.09/-4.57 (BC)

$$
49<-8.30 \div>-4.30 \quad-4.78 \quad 24 \quad-6.45 \div>-4.30 \quad-4.42 \quad 4 \quad 4.62 \div>-4.30 \quad-4.31
$$

Most sensitive cell lines $\left(\log \mathrm{GI}_{50} / \log \mathrm{TGI}\right)$ : RPMI-8226 -4.99/> -4.30 (L); NCI-H23 -4.97/-4.69; NCI-H522 -4.90/-4.56 (NSCLC); HCT-116 -4.94/-4.58 (CC); U251 -4,95/> -4.30 (CNS); SK-MEL5 <-8.30/-6.45 (M); OVCAR-8 -4.92/-4.47 (OC); ACHN -4.99/-4.69 (RC)

Most sensitive cell lines $\left(\log \mathrm{GI}_{50} / \log \mathrm{TGI}\right)$ : SR $-5.21 />-4.30(\mathrm{~L})$; NCI-H23 -5.09/-4.35 (NSCLC); U-251 -4.82/> -4.30 (CNS); LOX IMVI -5.93/-5.53 (M); UO-31 -6.08/-5.79 (RC)

Most sensitive cell lines $\left(\log \mathrm{GI}_{50} / \log \mathrm{TGI}\right)$ : CCRF-CEM -5.36/-5.41; RPMI8226 -6.17/-5.41; MOLT-4 -5.41/-4.00 (L); OVCAR-8 $-5.16 />-4.00(\mathrm{OC})$

$\begin{array}{cllllllll}8 & -6.05 \div-4.01 & -4.26 & 5 & -5.85 \div-4.01 & -4.09 & 3 & -5.02 \div-4.53 & -4.05 \\ 10 * & -7.71 \div-4.10 * & -4.17 * & 3 * & -5.51 \div-4.62 * & -4.05 * & 2 * & -4.28 \div-4.09 * & -4.01 *\end{array}$

Most sensitive cell lines $\left(\log \mathrm{GI}_{50} / \log \mathrm{TGI}\right)$ : CCRF-CEM $-6.06(5.92 *) />-4.00(-5.51 *)$; HL-60 (TB) $-6.53 /-5.70$; $\mathrm{K}-562-5.68(-5.27 *) />-4.00(>-4.00 *)$; MOLT-4 -6.52 $(-5.34 *) /-5.49(-4.62 *)$; SR $-6.51(-7.71 *) /-5.85(-4.90 *)(\mathrm{L})$; HOP-92 -4.74/-4.01 (NSCLC)

Most sensitive cell lines ( $\left.\log \mathrm{GI}_{50} / \log \mathrm{TGI}\right)$ : CCRF-CEM -4.83/-4.50; HL-60 (TB) -4.71/-4.33; MOLT-4 -4.96/-4.55; RPMI-8226 -4.68/-4.17; SR -5.15/-4.47 (L); HOP-62 -4.82/-4.36; HOP-92 -4.76/-4.38; NCI-H226 -4.80/-4.35 (NSCLC); SF-268 -4.98/-4.53, SF-539 -4.78/-4.41: SNB -4.88/-4.44; U251 -4.83/-4.53 (CNS); LOX-IMVI -4.81/-4.49 (M); OVCAR-4 -4.88/-4.19; SK-OV-3 -4.71/-4.29 (OC); SN-12C -4.77/-4.32; TK-10 -4.77/-4.37 (RC); MDA-MB-231/ATTC $-4.79 /-4.37$; HS-578T $-4.97 /-4.42$ (BC)

Most sensitive cell lines $\left(\log \mathrm{GI}_{50} / \log \mathrm{TGI}\right)$ : NCI NCI-H23 -4.58/-4.05 (NSCLC); HCT-116 -4.52/> -4.00 (CC); U251 -4.59/-4.16 (CNS); OVCAR-3 -4.51/-4.02 (OC); MCF-7 -5.55/> -4.00; MDA-MB-231/ATTC -4.63/-4.23 (BC) 
Continuation of Table

\begin{tabular}{|c|c|c|c|c|c|c|c|c|c|}
\hline 1 & 2 & 3 & 4 & 5 & 6 & 7 & 8 & 9 & 10 \\
\hline
\end{tabular}

$\begin{array}{lllllllll}12 & 11 & -6.71 \div-4.15 & -4.11 & 1 & - & -4.00 & 0 & -\end{array}$ Most sensitive cell lines $\left(\log \mathrm{GI}_{50} / \log \mathrm{TGI}\right)$ : CCRF-CEM $-6.71 />-4.00$ (L); U251 $-4.51 />-4.00$ (CNS)

$\begin{array}{llllllllll} & 20 & -4.66 \div>-4.06 & -4.13 & 2 & -4.43 \div-4.28 & -4.01 & 1 & -4.11 \div-4.00 & -4.00\end{array}$ Most sensitive cell lines $\left(\operatorname{logGI}{ }_{50} / \log \mathrm{TGI}\right)$ : $\mathrm{SR}-4,47 />-4.00(\mathrm{~L})$; NCI-H23 -4.50/> $-4.00 ; \mathrm{A}-498-4.75 /-4.43$ (NSCLC); MDA-MB-231/ATTC $-4.58 />-4.00 ;$ HS-578T $-4.66 /-4.28(\mathrm{BC})$

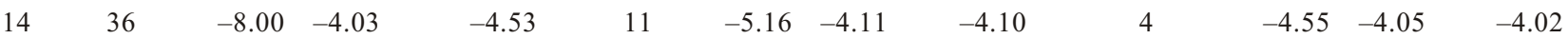

Most sensitive cell lines $\left(\log \mathrm{GI}_{50} / \log \mathrm{TGI}\right)$ : CCRF-CEM -4.87/> -4.00; MOLT-4 -5.60/> -4.00; RPMI-8226 -8.00/-5.16 (L); A549/ATTC -4.97/> -4.00; HOP-62 -4.90/-4.53. HOP-92 -5.27/-4.54; NCI-H226 -4.88/-4.33 (NSCLC);

HCT-116 -4.86/-4.18 (CC); M14 -4.84/> -4.00(M); 786-O -4.86/-4.45; ACHN -4.84/-4.54. SN12C -4.92/-4.46 (RC); MDA-MB-231/ATTC -5.34/-4.64; HS578T -4.98/-4.11 (BC)

$\begin{array}{llllllllll}15 & 1 & -8.00 \div-4.00 & -4.07 & 1 & -4.02 & -4.00 & - & -4.00\end{array}$

Most sensitive cell lines $\left(\log \mathrm{GI}_{50} / \log \mathrm{TGI}\right): \mathrm{HOP}-92<-8.00 /-4.02(\mathrm{NSCLC})$

$\begin{array}{llllllllll}16 & 48 & -5.55 \div-4.30 & -4.66 & 36 & -4.65 \div-4.30 & -4.26 & 18 & -4.33 \div-4.03 & -4.07\end{array}$

Most sensitive cell lines $\left(\log \mathrm{GI}_{50} / \log \mathrm{TGI}\right)$ : NCI-H522 -5.55/> -4.00 (NSCLC); U251 -4.91/-4.61(CNS); $\mathrm{PC}-3-4.87 /-4.52(\mathrm{PC})$

$\begin{array}{llllllllll}17 & 40 & -5.39 \div-4.20 & -4.41 & 13 & -4.30 \div-4.06 & -4.04 & 1 & -4.26 \div-4.00 & -4.00\end{array}$

Most sensitive cell lines $\left(\log \mathrm{GI}_{50} / \log \mathrm{TGI}\right)$ : CCRF-CEM -5.39/> -4.00; K-562 -5.04/> -4.00; MOLT-4 -5.33/> -4.00 (L)

$46-6.30 \div-4.06$

$-4.55 \quad 23-4.55 \div-4.04$

$-4.16$

8

$-4.27 \div-4.10$

$-4.03$

Most sensitive cell lines $\left(\log \mathrm{GI}_{50} / \log \mathrm{TGI}\right)$ : NCI-H23 -4.89/-4.55 (NSCLC); NCI-H522 -6.30/> -4.00

8

$-4.54 \div-4.16-4.04$

$-4.00$

$-4.00$

Most sensitive cell lines $\left(\log \mathrm{II}_{50} / \log \mathrm{TGI}\right)$ : MALME-3M $-4.54 />-4.00(\mathrm{M})$

55

$-4.78 \div-4.10$

$-4.56 \quad 31 \quad-4.39 \div-4.07$

$-4.13$

5

$-4.19 \div-4.03$

$-4.01$

Most sensitive cell lines $\left(\log \mathrm{GI}_{50} / \log \mathrm{TGI}\right)$ : SK-MEL-5 -4.78/-4.48; UACC-62 -4.74/-4.39 (M)

56

$-5.78 \div-4.87$

$-5.31$

$52-5.38 \div-4.02$

$-4.67$

22

$-4.80 \div-4.04$

$-4.12$

Most sensitive cell lines $\left(\log \mathrm{GI}_{50} / \log \mathrm{TGI}\right)$ : HL-60(TB) -5.66/-5.23; K-562 -5.43/-4.77 (L); NCI-H522 -5.78/-5.38 (NSCLC); KM12 -5.62/-5.18 (CC)

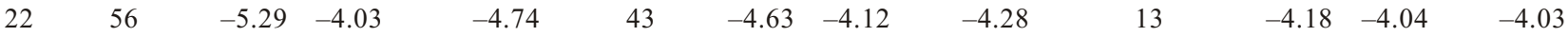

Most sensitive cell lines ( $\left.\log \mathrm{GI}_{50} / \log \mathrm{TGI}\right)$ : CCRF-CEM -5.18/-4.34; SR -5.18/-4.49(L); KM12 -5.11/-4.63 (CC); U251 $-5.10 /-4.61$ (CNS); PC-3 -5.29/-4.56 (PC)

$\begin{array}{llllllllll}23 & 21 & -4.90 \div-4.30 & -4.42 & 3 & -4.47 \div-4.30 & -4.31 & - & >-4.30 & -4.30\end{array}$

Most sensitive cell lines ( $\left.\log \mathrm{GI}_{50} / \log \mathrm{TGI}\right)$ : EKVX -4.83/-4.39; NCI-H23 -4.75/> -4.30 (NSCLC); SF-268 -4.86/-4.32 (CNS); LOX IMVI $-4.90 /-4.47$ (M) 


\begin{tabular}{c|c|c|c|c|c|c|c|c|c}
\hline 1 & 2 & 3 & 4 & 5 & 6 & 7 & 8 & 9 & 10 \\
\hline 24 & 47 & $-5.32 \div-4.16$ & -4.55 & 20 & $-4.35 \div-4.16$ & -4.13 & 8 & $-4.21 \div-4.02$ & -4.02
\end{tabular}

Most sensitive cell lines $\left(\log \mathrm{GI}_{50} / \log \mathrm{TGI}\right)$ : NCI-H522 -5.32/> -4.00 (NSCLC); SN12C -5.14/-4.08 (RC)

$\begin{array}{llllllllll}25 & 45 & -5.24 \div-4.44 & -4.88 & 32 & -4.88 \div-4.34 & -4.50 & 12 & -4.56 \div-4.34 & -4.33\end{array}$

Most sensitive cell lines $\left(\log \mathrm{GI}_{50} / \log \mathrm{TGI}\right)$ : SR $-5.16 /-4.72(\mathrm{~L})$; NCI-H322M -5.03/-4.53; NCI-H522 -5.01/-4.55 (NSCLC); UACC-62 -5.12/-4.84 (M); OVCAR-8 -5.14/-4.67 (OC); MDA-MB-435 -5.07/-4.74; MDA-MB-231/ATTC -5.16/-4.64; BT-549 $-5.24 /-4.88(\mathrm{BC})$

${ }^{\mathrm{a}}$ The value $>-4.00$ (or $>-4.30$ ) was excluded; *data of double assay; N 1, N 2, N 3 - number of sensitive cell lines; L - Leukemia; CC Colon Cancer; CNS - CNS Cancer; NSCLC - Non-Small Cell Lung Cancer; M - Melanoma; OC - Ovarian Cancer; BC - Breast Cancer; $\mathrm{RC}$ - Renal Cancer; PC - Prostate Cancer.
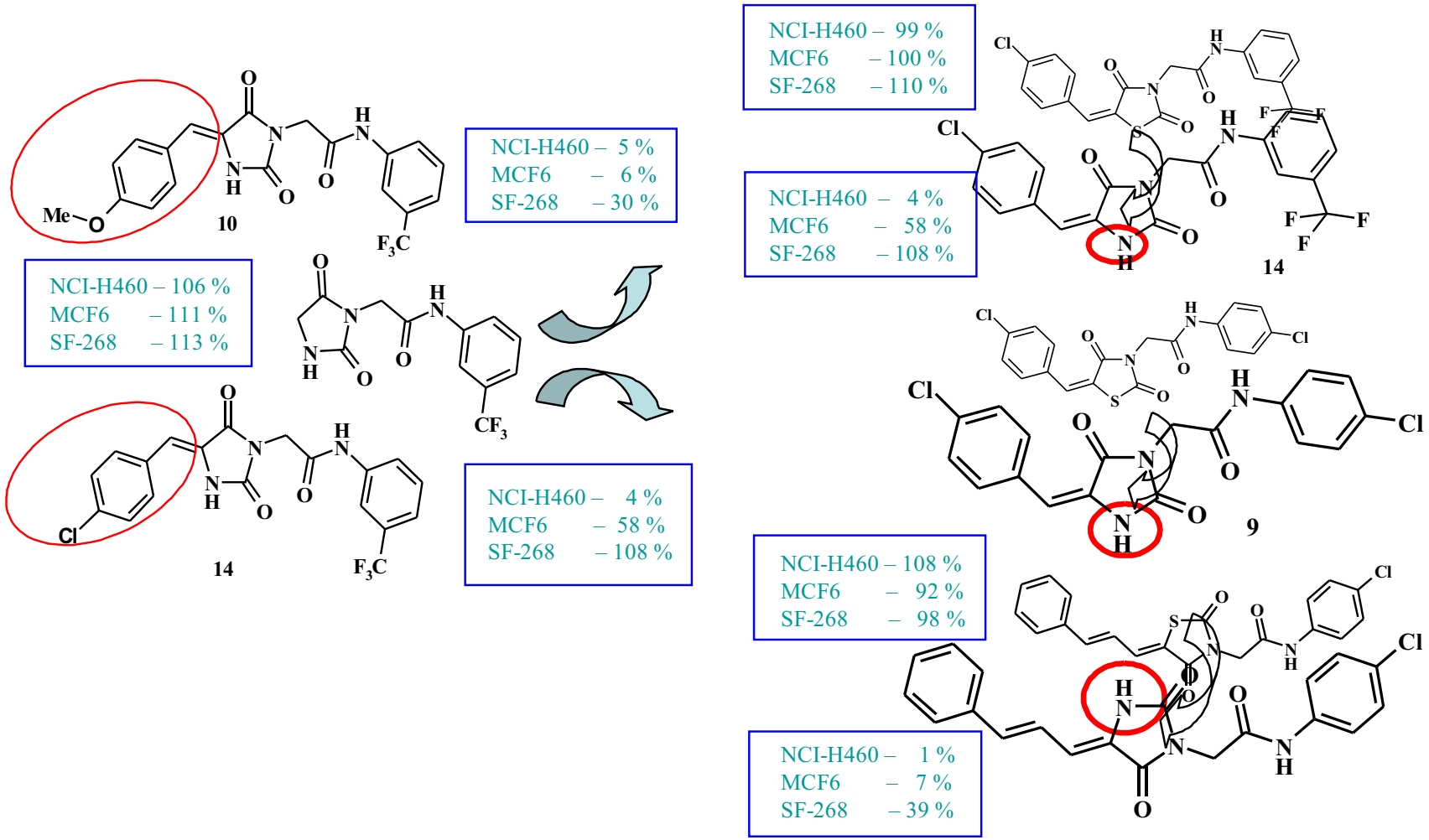

Fig. 4. Some structure-anticancer activity relationships aspects (in square grow percent for definite cancer cell lines)

served for 6-[5-(4-nitrobenzylidene)-4-oxo-2-thioxothiazolidin-3-yl]-hexanoic acid, 2-(5-isopropylidene2,4-dioxothiazolidin-3-yl)-N-(3-trifluoromethylphenyl)-acetamide influence on 786-O cell. Moreover, high sensitivity of all leukemia cell lines to studied 4azolidinone derivatives was detected.

As the next step 25 compounds were selected for advanced assays on 60 cell lines panel (at five 10-fold dilutions - concentrations ranging from $10^{-4}$ to $10^{-8} \mathrm{M}$ ) (Fig. 3). Tested compounds belonged to the following groups (5-aryl(heteryl)idenerhodanine-3-alkanemono(di)carboxylic acids, 5-arylidene-2,4-thiazolidinedione-3-alkanecarboxylic acids and 5-arylidene-2,4-imidazolidinedione-3-acetic acids) and showed different strength of anticancer activity - from practically absent to expressive action on all tested cell lines (Table). Obtained data allowed us to summarize some aspects of structure - anticancer activity relationships in testing row of 4-azolidinone derivatives. The presence of ylidene moiety in position 5 of core heterocycles plays 


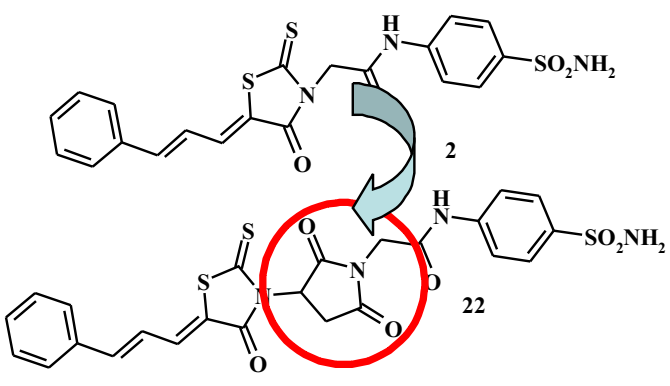

Combining of thiazolidinone and pyrrolidinedione cycles

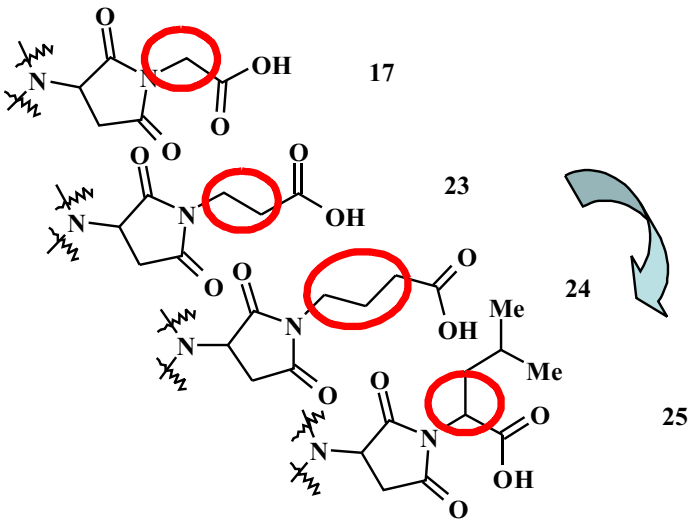

Desirable elongation or complicate of carbonic chain in $N$ of pyrrolidinedione moiety

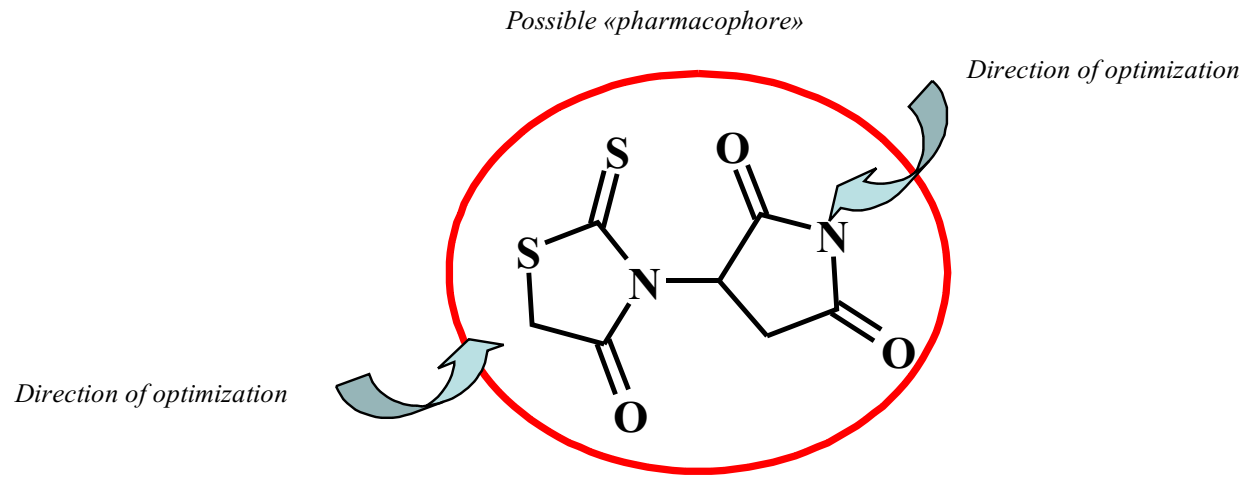

Fig. 5. Directions of lead optimization among 5-ylidenerhodanine-3-succinic acids derivatives

crucial role for achieving anticancer activity (Fig. 4). Presence of certain arylidene or phenylpropenylidene fragments is also desirable. This confirms our hypothesis about critical influence of the moiety in position 5 of core heterocycles on realization of biological effects, as it was previously established for another groups of 4-azolidinone and related heterocyclic systems derivatives $[1,28]$. Comparison of anticancer activity of free acids and their derivatives (namely amides) shows that latter are more active than corresponding acids as well as the results of [35]. $\mathrm{CF}_{3}$-Substituted anilines and sulfanilamide moieties are desirable as «privileged» fragments.

Comparison of anticancer activity of isosters of rhodanine and 2,4-thiazolidinedione derivatives or homologs of mentioned substances didn't allow us to establish any relation. However, substitution of S-atom of thiazolidinone ring for $\mathrm{N}$-atom (transfer from 2,4- thiazolidinedione to 2,4-imidazolidinedione) contributes to increase in anticancer activity and appearing of selectivity. Hydantoin-3-acetic acids derivatives (14, $8,9,11,12)$ possess the distinct selective influence on Leukemia cell lines comparing to the other groups of cancer cell lines. This fact allows us to interpret the 5arylidene-2,4-imidazolidinedione-3-acetic acids amides as lead-compounds in search of antileukemic agents. In addition, mentioned group is more active in comparison to other 2,4-thia(imida)zolidinedione derivatives [28].

Analysis of anticancer activity data of 5-ylidenerhodanine-3-succinic acids derivatives allowed to summarize some structure-activity correlations (Fig. 5). Modification of free dicarboxylic acids to their diamides caused increase in anticancer activity, which was the most prominent for cyclic imides. Based on the interpretation of obtained data 3-(4-oxo-2-thioxothia- 
zolidine-3-yl)-pyrrolidine-2,5-dione fragment was assumed as possible pharmacophore within investigated samples row [29]. Consequently, we showed the directions of this fragment chemical modification aimed at structure optimization, namely: position $\mathrm{C} 5$ of rhodanine cycle and $\mathrm{N}$-atom of pyrrolidine.

Conclusion. The present study describes in vitro anticancer activity of new 5-ylidene-4-thiazolidinone3-alkanecarboxylic acids derivatives. The series of active compounds with high activity and/or selectivity levels were selected. Some aspects of structure-anticancer activity relationships were determined and structure design directions were proposed. 5-Arylidenehydantoin-3-acetic acids derivatives were identified as a new class of potent antileukemic agents. Possible pharmacophore scaffold of 5-ylidenerhodanine-3-succinic acids derivatives was suggested.

Acknowledgements. We are grateful to Dr. V. L. Narayanan from Drug Synthesis and Chemistry Branch, National Cancer Institute, Bethesda, MD, USA, for in vitro evaluation of anticancer activity.

\section{Д. В. Камінський, Р. Б. Лесик}

Взаємозв'язок «структура-протиракова активність» в ряду 4азолідон-3-карбонових кислот та їхніх похідних

Резюме

Мета даного дослідження полягала у вивченні протиракової активності 4-азолідон-3-карбонових кислот та їхніх похідних, встановленні особливостей взаємозв'язку «структура-активність». Методи. Органічний синтез, спектральні методи, скринінг протипухлинної активності (US NCI-методології, Developmental Therapeutic Program). Результати. Представлено результати тестування in vitro протиракової активності нових похідних 4-азолідон-3-алканкарбонових кислот. Виділено високоактивні сполуки, які належать до похідних 5-ариліден-2,4-тіа(іміда)золідон-3-алканкарбонових кислот та 5-арил(гетерил)іденроданін-3-сукцинатних кислот. Встановлені закономірності залежності «структура-активність» дозволяють окреслити напрямки оптимізачії структурлідерів і ідентифікувати молекулярні фрагменти для дизайну потенційних протиракових агентів на основі 4-азолідонового скаффолду. Аміди 5-ариліденгідантоїн-3-оитових кислот визначено як новий клас протилейкемічних агентів. Для 5-іліденроданін-3-сукцинатних кислот ідентифіковано ймовірний фармакофор. Висновки. Одержано низку активних сполукз високим рівнем протиракової активності та/або селективності. Запропоновано напрямки дизайну структури потенційних протиракових агентів на основі встановлених закономірностей «структура-активність».

Ключові слова: 4-азолідон-3-карбонові кислоти, протиракова активність, взаємозв'язок «структура-активність».

\section{Д. В. Каминский, Р. Б. Лесык}

Взаимосвязь «структура-противоопухолевая активность» в ряду 4-азолидон-3-карбоновых кислот и их производных

Резюме

Цель данного исследования состояла в изучении противоопухолевой активности 4-азолидон-3-карбоновых кислот и их производных, а также в установлении некоторых особенностей взаимосвязи «структура-активность». Методы. Органический синтез, спектральные методы, скрининг противоопухолевой активности (US NCI-методология, Developmental Therapeutic Program). Результаты. Представлены результаты тестирования in vitro противоопухолевой активности новых производных 4-азолидон-3-аланкарбоновых кислот. Отобраны наиболее активные соединения, которые относятся к производным 5-арилиден-2,4-тиа(имида)золидон-3-алканкарбоновых кислот и 5-арил(гетерил)иденроданин-3-сукцинатных кислот. На основании выявленных закономерностей взаимосвязи «структура-активность» определены направления оптимизации структур-лидеров, идентифицированы молекулярные фрагменты для дизайна потенцииальных противоопухолевых агентов на основании 4-азолидонового скаффолда. Амиды 5арилиденгидантоин-3-уксусных кислот рассматриваются как новый класс противолейкемических агентов. Для ряда 5-илиденроданин-3-сукцинатных кислот установлен вероятный фармакофор. Выводы. Выделен ряд активных соединений с высоким уровнем противоопухолевой активности и/или селективности. Предложены направления дизайна структуры потенциальных противоопухолевых агентов на основе установленных закономерностей «структура-активность».

Ключевые слова: 4-азолидон-3-карбоновые кислоты, противоопухолевая активность, взаимосвязь «структура-активность».

\section{REFERENCES}

1. Lesyk R. B., Zimenkovsky B. S. 4-Thiazolidones: Centenarian history, current status and perspectives for modern organic and medicinal chemistry // Curr. Org. Chem.-2004.-8, N 16.-P. 1547-1577.

2. Prabhakar Y. S., Solomon V. R., Gupta M. K., Katti S. B. QSAR studies on thiazolidines: biologically privileged scaffold // Top. Heterocycl. Chem.-2006.-4.-P. 161-249.

3. Ottana R., Carotti S., Maccari R., Landini I., Chiricosta G., Caciagli B., Vigorita M. G., Mini E. In vitro antiproliferative activity against human colon cancer cell lines of representative 4-thiazolidinones. Part I // Bioorg. Med. Chem. Lett.2005.-15, N 17.-P. 3930-3933.

4. Shih M. H., Ke F. Y. Synthesis and evaluation of antioxidant activity of sydnonyl substituted thiazolidinone and thiazoline derivatives // Bioorg. Med. Chem.-2004.-12, N 17.-P. 46334643.

5. Kesel A. S., Sonnenbicher I., Polborn K., Gurtler L., Klinkert W. E. F., Modolell M., Nussler A. K., Oberthur W. A new antioxidative vitamine B6-analogue modulates pathophysiological cell proliferation and demage // Bioorg. Med. Chem.1999.-7, N 2.-P. 359-367.

6. Avendano C., Menendez J. C. Medicinal chemistry of anticancer drugs.-Amsterdam: Elsevier, 2008. -400 p. 
7. Liu W. J., Bulgaru A., Haigentz M., Stein C. A., Perez-Soler $R$., Mani $S$. The BCL2-family of protein ligands as cancer drugs: the next generation of therapeutics // Curr. Med. Chem. Anti Cancer Agents.-2003.-3, N 3.-P. 217-223.

8. Degterev A., Lugovskoy A., Cardone M., Mulley B., Wagner G., Mitchison T., Yuan J. Identification of small-molecule inhibitors of interaction between the BH3 domain and Bcl-xl // Nat. Cell Biol.-2001.-3, N 2.-P. 173-182.

9. Lugovskoy A. A., Degterev A. I., Fahmy A. F., Zhou P., Gross J. D., Yuan J., Wagner G. A Novel approach for characterizing protein ligand complexes: molecular basis for specificity of small-molecule Bcl-2 inhibitors // J. Am. Chem. Soc.2002.-124, N 7.-P. 1234-1240.

10. Xing C., Wang L., Tang X. H., Sham Y.Y. Development of selective inhibitors for anti-apoptotic Bcl-2 proteins from BHI$1 / /$ Bioorg. Med. Chem.-2007.-15, N 5.-P. 2167-2176.

11. Cutshall N. S., O'Day C., Prezhdo M. Rhodanine derivatives as inhibitors of JSP-1// Bioorg. Med. Chem. Lett.-2005.-15, N 14.-P. 3374-3379.

12. Look G. C., Shuilck J. R., Holmes Ch. P., Chinn J. P., Gordon E. M., Gallop M. A. The identification of cyclooxygenase-1 inhibitors from 4-thiazolidinone combinatorial library // Bioorg. Med. Chem. Lett.-1996.-6, N 6.-P. 707-712.

13. Lee J., Kim J., Koh J. S., Chung H. H., Kim K. H. Hydantoin derivatives as non-peptidic inhibitors of Ras farnesyl transferase // Bioorg. Med. Chem. Lett.-2006.-16, N 7.-P. 19541956.

14. Mazieres J., Pradines A., Favre G. Perspectives on farnesyl transferase inhibitors in cancer therapy // Cancer Lett.2004.-206, N 2.-P. 159-164.

15. Zhao L., Huang $W$., Liu H., Wang L., Zhong W., Xiao J., Hu $Y$., Li S. FK506-binding protein ligands: structure-based design, synthesis, and neurotrophic/neuroprotective properties of substituted 5,5-dimethyl-2-(4-thiazolidine) carboxylates // J. Med. Chem.-2006.-49, N 14.-P. 4059-4071.

16. Liuqing W., Gan X., Zhong J., Alliston K. R., Groutas W. C. Noncovalent inhibitors of human leukocyte elastase based on the 4-imidazolidinone scaffold // Bioorg. Med. Chem.2003.-11, N 23.-P. 5149-5153.

17. Carmi C., Cavazzoni A., Zuliani V., Lodola A., Bordi F., Plazzi P. V., Alfieri R. R., Petronini P. G., Mor M. 5-Benzylidene-hydantoins as new EGFR inhibitors with antiproliferative activity // Bioorg. Med. Chem. Lett.-2006.-16, N 15.P. 4021-4025.

18 Rajic Z., Zorc B., Raic-Malic S., Ester K., Kralj M., Pavelic K., Balzarini J., Clercq E. D., Mintas M. Hydantoin derivatives of L- and D-amino acids: synthesis and evaluation of their antiviral and antitumoral activity // Molecules.-2006.-11, N 11.-P. 837-848.

19. Gibbs J. B., Oliff A. The potential of farnesyltransferase inhibitors as cancer chemotherapeutics // Ann. Rev. Pharmacol. Toxicol.-1997.-37.-P. 143-166.

20. Haluska P., Dy G. K., Adjei A. A. Farnesyl transferase inhibitors as anticancer agents // Eur. J. Cancer.-2002.-38, N 13.P. $1685-1700$.

21. Lv P. C., Zhou C. F., Chen J., Liu P. G., Wang K. R., Mao W. J., Li H. Q., Yang Y., Xiong J., Zhu H. L. Design, synthesis and biological evaluation of thiazolidinone derivatives as potential EGFR and HER-2 kinase inhibitors // Bioorg. Med. Chem.-2010.-18, N 1.-P. 314-319.

22. Pevarello P., Brasca M. G., Orsini P., Traquandi G., Longo A., Nesi M., Orzi F., Piutti C., Sansonna P., Varasi M., Cameron A., Vulpetti A., Roletto F., Alzani R., Ciomei M., Albanese
C., Pastori W., Marsiglio A., Pesenti E., Fiorentini F., Bischoff J. R., Mercurio C. 3-Aminopyrazole inhibitors of CDK2/cyclin A as antitumor agents. 2. Lead optimization // J. Med. Chem.-2005.-48, N 8.-P. 2944-2956.

23. Dayam R., Aiello F., Deng J., Wu Y., Garofalo A., Chen X., Neamati $N$. Discovery of small molecule integrin $\alpha_{v} \beta_{3}$ antagonists as novel anticancer agents // J. Med. Chem.-2006.49, N 15.-P. 4526-4534.

24. Pat. US2003/0119894, IPC A 61K31/404, N 09/9106291. Methods for treatment of cancer or neoplastic disease and for inhibiting growth of cancer cells and neoplastic cellsmurthy / M. S. R. Murthy, G. C. Shore, J. Bajorath, F. L. Stahura // Publ. 26.06.2003.

25. Teng X., Degterev A., Jagtap P., Xing X., Choi S., Denu R., Yuan J., Cuny G. D. Structure-activity relationship study of novel necroptosis inhibitors // Bioorg. Med. Chem. Lett.2005.-15, N 22.-P. 5039-5044.

26. Lesyk R., Zimenkovsky B., Lukyanchuk V., Atamanyuk D., Vovk $O$. Chemistry and pharmacology of 4-thiazolidone derivatives // Ann. Polish Chem. Soc.-2003.-2.-P. 293-298.

27. Zimenkovsky B. S., Lesyk R. B. Purposeful synthesis of biological active compound base on 4-azolidinone // J. Org. Pharm. Chem.-2003.-1, N 1-2.-P. 24-30 (in Ukrainian).

28. Kaminskyy D., Zimenkovsky B., Lesyk R. Synthesis and in vit$r o$ anticancer activity of 2,4-azolidinedione-acetic acids derivatives // Eur. J. Med. Chem. -2009.-44, N 9.-P. 3627-3636.

29. Kaminskyy D. V., Roman O. M., Atamanyuk D. V., Lesyk R. B. 5-Ylidene-2-thioxo-4-thiazolidinone-3-succinic acids and their derivatives: synthesis, anticancer activity, QSAR-analysis // J. Org. Pharm. Chem.-2006.-4, N 1(13).-P. 41-48.

30. Kaminskyy D. V., Lesyk R. B. Synthesis and biological activity of 4-thiazolidinone-3-acetic acids derivatives // Farmacevt. Zhur. (Kyiv).-2008.-3.-P. 70-78.

31. Monks A., Scudiero D., Skehan P., Shoemaker R., Paull K., Vistica D., Hose C., Langley J., Cronise P., Vaigro-Wolff A., Gray-Goodrich M., Campbell H., Mayo J., Boyd M. Feasibility of a high-flux anticancer drug screen using a diverse panel of cultured human tumor cell lines // J. Nat. Cancer Inst.-1991.-83, N 11.-P. 757-766.

32. Boyd M. R., Paull K. D. Some practical considerations and applications of the national cancer institute in vitro anticancer drug discovery screen // Drug Develop. Res.-1995.-34, N 2.P. 91-109.

33. Boyd M. R. Anticancer drug development guide: preclinical screening, clinical trials, and approval in: cancer drug discovery and development / Ed. A. Teicher.-Totowa: Humana Press, 1997.-P. 23-43.

34. Shoemaker R. H. The NCI60 human tumour cell line anticancer drug screen // Nat. Rev. Cancer.-2006.-6, N 10.-P. 813823.

35. Gududuru V., Hurh E., Dalton J. T., Miller D. D. Synthesis and antiproliferative activity of 2-aryl-4-oxo-thiazolidin-3yl-amides for prostate cancer // Bioorg. Med. Chem. Lett.2004.-14, N 21.-P. 5289-5293.

UDC 615.012.1.076:547.789.1 Received 10.01.10 